\title{
Structural disconnectivity and the risk of dementia in the general population
}

Lotte G.M. Cremers, PhD, Frank J. Wolters, MD, Marius de Groot, PhD, M. Kamran Ikram, PhD, Aad van der Lugt, PhD, Wiro J. Niessen, PhD, Meike W. Vernooij, PhD,* and M. Arfan Ikram, PhD*

Neurology ${ }^{\circledR}$ 2020;95:e1528-e1537. doi:10.1212/WNL.0000000000010231

\section{Correspondence}

Dr. Ikram

m.a.ikram@erasmusmc.nl

\section{Abstract}

\section{Objective}

The disconnectivity hypothesis postulates that partial loss of connecting white matter fibers between brain regions contributes to the development of dementia. Using diffusion MRI to quantify global and tract-specific white matter microstructural integrity, we tested this hypothesis in a longitudinal population-based study.

\section{Methods}

Global and tract-specific fractional anisotropy (FA) and mean diffusivity (MD) were obtained in 4,415 people without dementia (mean age 63.9 years, $55.0 \%$ women) from the prospective population-based Rotterdam Study with brain MRI between 2005 and 2011. We modeled the association of these diffusion measures with risk of dementia (follow-up until 2016) and with changes on repeated cognitive assessment after on average 5.4 years, adjusting for age, sex, education, macrostructural MRI markers, depressive symptoms, cardiovascular risk factors, and $A P O E$ genotype.

\section{Results}

During a median follow-up of 6.8 years, 101 participants had incident dementia, of whom 83 had clinical Alzheimer disease (AD). Lower global values of FA and higher values of MD were associated with an increased risk of dementia (adjusted hazard ratio [95\% confidence interval (CI) ] per SD increase for MD 1.79 [1.44-2.23] and FA 0.65 [0.52-0.80]). Similarly, lower global values of FA and higher values of MD related to more cognitive decline in people without dementia (difference in global cognition per SD increase in $\mathrm{MD}$ [95\% CI] was $-0.04[-0.07$ to $-0.01])$. Associations were most profound in the projection, association, and limbic system tracts.

\section{Conclusions}

Structural disconnectivity is associated with an increased risk of dementia and more pronounced cognitive decline in the general population.

*These authors contributed equally to this work.

From the Departments of Radiology and Nuclear Medicine (L.G.M.C., F.J.W., M.d.G., A.v.d.L., W.J.N., M.W.V.), Epidemiology (L.G.M.C., F.J.W., M.d.G., M.K.I., M.W.V., M.A.I.), Neurology (F.J.W., M.K.I., M.A.I.), and Medical Informatics (M.d.G., W.J.N.), Erasmus MC, Rotterdam; and Department of Imaging Physics, Faculty of Applied Sciences (W.J.N.), Delft University of Technology, Delft, the Netherlands.

Go to Neurology.org/N for full disclosures. Funding information and disclosures deemed relevant by the authors, if any, are provided at the end of the article. 


\section{Glossary}

AD = Alzheimer disease; BMI = body mass index; CES-D score = Center for Epidemiologic Studies Depression Scale; DSMIII-R = Diagnostic and Statistical Manual of Mental Disorders, 3rd edition, revised; FA = fractional anisotropy; FLAIR = fluidattenuated inversion recovery; GMS = Geriatric Mental Schedule; HDL = high-density lipoprotein; HR = hazard ratio; ICV = intracranial volume; $\mathbf{M D}=$ mean diffusivity; $\mathbf{M M S E}=$ Mini-Mental State Examination; WMH = white matter hyperintensity.

Dementia is among the leading causes of death and disability worldwide, and its socioeconomic burden on society will continue to increase as the number of persons with dementia is predicted to nearly triple to 131 million in 2050. ${ }^{1}$ Effective preventive and curative interventions are urgently needed, but their development and timely application is hampered by incomplete understanding of pathophysiology, lack of markers that can identify changes in the very early, subclinical stages of disease, and lack of prognostic markers. Subclinical brain changes are thought to occur years, if not decades, prior to onset of clinical symptoms, ${ }^{2}$ which is beyond the scope of currently applied subclinical macrostructural imaging markers of neurodegeneration, such as hippocampal volume and presence of white matter hyperintensities (WMHs). Despite advances in measurement of amyloid and tau, these measurements come at high cost and provide incomplete answers to prediction of dementia in the presence of a multitude of pathologies at old age. ${ }^{3}$ In particular, when selecting individuals in the community for further screening or trial inclusion, imaging tools are valuable to improve prognostic precision beyond clinical characteristics. ${ }^{4}$

One of the recent insights in dementia is that brain damage can lead to disruption of brain networks, so called disconnectivity. ${ }^{5-7}$ Disconnectivity, which can be investigated using diffusion MRI, seems to occur prior to changes in conventional structural MRI markers such as WMHs load in dementia, ${ }^{8}$ and is thought to reflect early cerebral white matter damage. ${ }^{9,10}$ Disconnectivity is more pronounced in patients with dementia compared to healthy controls, ${ }^{11,12}$ and relates to more rapid cognitive decline in patients with Alzheimer disease (AD) ${ }^{13}$ In 4 longitudinal studies from 2 clinical cohorts of patients with small vessel disease, network disruption was related to accelerated decline in psychomotor speed and an increased risk of dementia. ${ }^{14-17}$ However, patients with substantial small vessel disease on MRI represent a minority of the individuals at high risk of dementia in the community, and it remains undetermined whether prior findings extend to the wider population without severe small vessel disease, prior TIA, or stroke. In addition, study in persons with and without small vessel disease may better determine the effect of disconnectivity on dementia, above and beyond the burden of, for example, WMHs.

We aimed to determine the association of global and tractspecific disconnectivity with dementia and cognitive decline in a population-based setting.

\section{Methods}

\section{Standard protocol approvals, registrations, and patient consents}

The Rotterdam Study has been approved by the medical ethics committee according to the Population Study Act Rotterdam Study, executed by the Ministry of Health, Welfare and Sports of the Netherlands. All participants gave written informed consent.

\section{Study population}

This study was embedded within the Rotterdam Study, a population-based cohort study including participants 45 years and older living in Ommoord, a suburb of Rotterdam. ${ }^{18}$ The study started in 1990 with 7,983 participants and was extended with 3,011 participants in 2000 and with 3,932 participants in 2006. Participants were examined at baseline with a home interview and an extensive set of examinations in the research center. Follow-up examinations were repeated every 3-4 years. All participants were continuously monitored through electronic linkage of the study database with their own medical records. All details of the study have been described previously. ${ }^{18}$ From 2005 onwards, MRI scanning was implemented in the core protocol. Between 2005 and 2011, 5,715 participants without contraindications for MRI (metal implants, pacemaker, claustrophobia) were eligible for scanning, of whom 4,888 (86\%) underwent a multisequence MRI acquisition of the brain, and 4,813 (98\%) participants completed the diffusion-weighted sequences. We excluded 245 individuals due to technical scanning issues, e.g., failed segmentations, as well as 38 participants with prevalent dementia and 100 participants with insufficient dementia screening at baseline, resulting in a study sample of 4,430 individuals. Of these individuals, 4,317 persons had detailed cognitive assessment at baseline and 3,402 (79\%) had repeated assessment during follow-up examination after on average 5.4 (SD 0.6) years.

\section{MRI acquisition and processing}

Multisequence MRI was performed on a 1.5T MRI scanner (GE [Chalfont St. Giles, UK] Signa Excite). The imaging protocol has been described extensively elsewhere. ${ }^{19}$ The conventional scan protocol consisted of a T1-weighted image, a T2-weighted fluid-attenuated inversion recovery (FLAIR) sequence, and a proton density-weighted image.

Scans were spatially coregistered using rigid registration. Scans were segmented with an automated tissue segmentation approach into gray matter, white matter, CSF, and background tissue, ${ }^{20,21}$ followed by WMH segmentation based on the tissue 
segmentation and the FLAIR image. ${ }^{22}$ Supratentorial intracranial volume (ICV), to correct for head size, was estimated by summing total gray and white matter and CSF volumes. ${ }^{21}$ We visually assessed the presence of infarcts on conventional MRI sequences, and in case of involvement of cortical gray matter, we classified these as cortical infarcts.

\section{Diffusion MRI processing and tractography}

For diffusion MRI, we performed a single-shot, diffusionweighted spin echo echoplanar imaging sequence. Maximum b-value was $1,000 \mathrm{~s} / \mathrm{mm}^{2}$ in 25 noncollinear directions; 3 volumes were acquired without diffusion weighting (b-value $\left.=0 \mathrm{~s} / \mathrm{mm}^{2}\right)$. All diffusion data were preprocessed using a standardized pipeline. ${ }^{23}$ In short, eddy current and head motion correction were performed on the diffusion data. The resampled data were used to fit diffusion tensors to compute mean fractional anisotropy (FA) and mean diffusivity $(\mathrm{MD})$ in the normal-appearing white matter, through combination with the tissue segmentation. The diffusion data were also used to segment white matter tracts using a diffusion tractography approach described previously. ${ }^{24}$ The tract-specific analysis was performed incorporating all voxels of the tract anatomy, both normal-appearing white matter voxels and voxels containing WMHs. Tractography was performed in native space, using standard space seed, target, stop, and exclusion masks as described previously. ${ }^{24}$ Tractography was performed with PROBTRACKX, a Bayesian framework for white matter tractography, available in FSL (version 4.1.4). Protocols for identifying 15 white matter tracts were defined as described previously and were made available as the autoPTX plugin for FSL (version 0.1.1). The reproducibility of the tractography was $87 \%$, as previously shown. ${ }^{24}$ The amount of seed points was variable across tracts to achieve a robust sampling of all tracts investigated. The ball and stick diffusion model (BedpostX) estimation and tractography algorithm were run with default settings. We segmented 15 different white matter tracts ( 12 bilateral, 3 singular) and obtained mean FA and MD in these tracts, with subsequent combination of left and right measures. ${ }^{24}$ In general, lower FA and higher MD values are considered indicative of lower microstructural integrity and as such reflecting disconnectivity. Missing data for tract-specific measurements due to tractography or segmentation failures were limited to $33-78$ participants $(0.8 \%-1.8 \%)$ per tract. Tracts were categorized, based on anatomy or presumed function, into brainstem tracts (middle cerebellar peduncle, medial lemniscus), projection tracts (corticospinal tract, anterior thalamic radiation, superior thalamic radiation, posterior thalamic radiation), association tracts (superior longitudinal fasciculus, inferior, longitudinal fasciculus, inferior fronto-occipital fasciculus, uncinated fasciculus), limbic system tracts (cingulate gyrus part of cingulum, parahippocampal part of cingulum and fornix), and callosal tracts (forceps major, forceps minor). ${ }^{24}$

We obtained tract volumes and tract $\mathrm{WMH}$ volumes by combining the tissue and tract segmentations. Tract-specific
WMH volumes were natural-log transformed, to account for their skewed distribution.

Between February 2007 and May 2008, an erroneous swap of the phase and frequency encoding directions for the diffusion acquisition led to a mild ghosting artifact, which was addressed by adjustment in the analysis. ${ }^{24}$ There was only partial coverage of one of the brainstem tracts (medial lemniscus) due to incomplete coverage of the cerebellum in the field of view, and we used alternative seed masks for tractography and adjustment in the model to overcome this problem. $^{24}$

\section{Dementia screening and surveillance}

All participants were screened for dementia at baseline and during subsequent center visits using the Mini-Mental State Examination (MMSE) and the Geriatric Mental Schedule (GMS) organic level. ${ }^{25}$ Participants with an MMSE score $<26$ or a GMS score $>0$ underwent further cognitive examination and informant interview, including the Cambridge Examination for Mental Disorders of the Elderly. In addition, the entire cohort was under continuous surveillance for dementia through electronic linkage of the study database with medical records from general practitioners and the regional institute for outpatient mental health care. Clinical neuroimaging was used when required for dementia subtype diagnosis. A consensus panel led by a consultant neurologist established the final diagnosis in accordance with standard criteria for dementia (DSM-III-R) and AD (National Institute of Neurological and Communicative Disorders and Stroke-Alzheimer's Disease and Related Disorders Association). Follow-up until January 1, 2016, was virtually complete ( $96 \%$ of potential person years). Participants were censored at date of dementia diagnosis, death, loss to follow-up, or January 1, 2016, whichever came first.

\section{Assessment of cognitive function}

During center visits, all participants underwent routine cognitive assessment comprising a word fluency test (number of animal species within 1 minute), 15-word learning test (immediate and delayed recall of 15 items), letter-digit substitution task (number of correct digits in 1 minute), Stroop test (error-adjusted time in seconds taken for completing the reading, color naming, and interference tasks), and the Purdue Pegboard task for manual dexterity. ${ }^{21}$ To obtain a composite measure of test performance, we calculated the G-factor by principal component analysis, ${ }^{21}$ which explained $49 \%-54 \%$ of variance in cognitive test scores at each examination round in our population. For each participant, $Z$ scores were calculated for each test separately, by dividing the difference between individual test score and population mean by the population SD. Scores for the Stroop tasks were inverted such that higher scores indicated better performance.

\section{Other measurements}

Information on smoking habits, educational attainment, and use of antihypertensive and lipid-lowering medication was 
ascertained at baseline by structured questionnaires. Blood pressure was measured twice in sitting position using a random-zero sphygmomanometer and the mean of 2 readings was used in the analyses. Total serum cholesterol and high-density lipoprotein (HDL) cholesterol were determined in fasting blood samples. Presence of type 2 diabetes at baseline was determined on the basis of fasting serum glucose level $(\geq 7.0 \mathrm{mmol} / \mathrm{L})$ or, if unavailable, nonfasting serum glucose level $(\geq 11.1 \mathrm{mmol} / \mathrm{L})$ or the use of antidiabetic medication. ${ }^{26}$ Body mass index (BMI) was calculated, dividing weight in kilograms by the squared height in meters. History of stroke was assessed by interview, and verified in medical records, and participants were continuously monitored for incident stroke through computerized linkage of medical records from general practitioners and nursing home physicians with the study database. We used the validated Dutch version of the Center for Epidemiologic Studies Depression Scale (CES-D) for assessment of depressive symptoms. ${ }^{27}$

APOE genotype was determined using PCR on coded DNA samples (original cohort) and using a bi-allelic TaqMan assay (rs7412 and rs429358; expansion cohort). In 179 participants with missing APOE status from this blood sampling, genotype was determined by genetic imputation (Illumina $610 \mathrm{~K}$ and 660K chip; imputation with Haplotype Reference Consortium reference panel [v1.0] with Minimac 3).

\section{Statistical analysis}

Analyses included all eligible participants, with the exception of 15 participants whose diffusion measures deviated $>7$ SDs from the mean, leaving 4,415 participants for analysis. We used Cox proportional hazard models to determine the etiologic association of global and tract-specific diffusion MRI measures (FA and MD) with incident dementia. The proportional hazard assumption was met. We assessed risk of dementia per SD increase in FA and $\mathrm{MD}$. We repeated the analyses (1) for AD only, (2) after excluding participants with prevalent stroke while censoring at time of incident stroke, (3) excluding persons with MRI-defined, subclinical cortical infarcts at baseline, and (4) stepwise excluding the first 5 years of follow-up from the analysis.

We then determined the association of global and tractspecific diffusion MRI measures with change in cognitive performance using linear regression models. Cognitive test scores at follow-up were adjusted for baseline cognitive test results. These analyses were repeated after exclusion of all participants who developed dementia during follow-up.

All models were adjusted for age, sex, education, ICV, white matter volume, and the log-transformed volume of WMHs and the correction for swapping gradients and varying field of view (model I), and in addition for education, depressive symptoms (CES-D score), and cardiovascular risk factors (systolic blood pressure, diastolic blood pressure, antihypertensive medication, serum cholesterol, HDL cholesterol, lipid-lowering medication, diabetes, smoking, and BMI) and APOE $\varepsilon 4$ allele carriership (model II). We adjusted for both ICV and white matter volume to take both developmental and neurodegenerative markers into account.

For the tract-specific analyses, we corrected the $p$ value ( $\alpha$ level of 0.05) for multiple comparisons with the number of independent tests on the basis of the variance of the eigenvalues of the correlation matrix of all 30 variables used in the main analysis (i.e., FA and MD for the 15 tracts). The following formula was used: $\mathrm{M}_{\mathrm{eff}}=1+(\mathrm{M}-1)(1-\mathrm{var}$ $\left.\left(\lambda_{\text {obs }}\right) / M\right)$, in which $M$ is the number of variables, $\lambda_{\text {obs }}$ is the variance of the eigenvalues of the correlation matrix, and $\mathrm{M}_{\mathrm{eff}}$ is the number of independent variables. ${ }^{28,29}$ This resulted in an $\mathrm{M}_{\text {eff }}$ of 17.45 , which then, using the Šidák formula $\left(\alpha\right.$ sidak $\left.=1-\left((1-\alpha)\left(1 / \mathrm{M}_{\mathrm{eff}}\right)\right)\right)$, translated into a significance level of $p<0.0029$ for the tract-specific analyses with dementia as outcome. ${ }^{28}$

For the analyses assessing global diffusion MRI measures with the separate cognitive tests as outcome, the above-mentioned method generated a significance level of $p<0.008$.

All analyses were carried out using SPSS Statistics 21.0 (IBM, Armonk, NY) or R version 3.0.3 (packages GenABEL, survival, stargazer, and data.table).

\section{Data availability}

Requests for anonymized data will be considered by the corresponding author.

\section{Results}

Table 1 presents the baseline characteristics of the study population. Mean age of the 4,415 participants was 63.9 years ( $\mathrm{SD} \pm 11.1$ years), and $55.0 \%$ were women. During a median follow-up of 6.8 years (interquartile range 5.8-8.0 years), 101 persons developed dementia, of whom 83 had $\mathrm{AD}$.

Lower microstructural integrity, reflected in lower values of global FA and higher values of global MD, was associated with a higher risk of dementia (fully adjusted hazard ratio [HR] [95\% confidence interval] per SD increase in FA 0.65 [0.52-0.80] and for MD 1.79 [1.44-2.23]; table 2). Results were similar for clinical $\mathrm{AD}$ only, and unaltered after excluding participants with prevalent stroke while censoring at time of incident stroke, or excluding participants with subclinical MRI-defined cortical infarcts (table 2). Stepwise exclusion of the first 5 years of follow-up from the analysis did not alter the risk estimates (figure 1). Further adjustment for hippocampal volume mildly attenuated the effect estimates (MD [HR] for all-cause dementia 1.67 [1.33-2.10], and for clinical AD 1.58 [1.23-2.04]) (data available from Dryad, table e-1, 10.5061/dryad.7wm37pvpq). 
Table 1 Population characteristics

\begin{tabular}{|c|c|}
\hline Characteristics & $\begin{array}{l}\text { Values } \\
\text { (total } n=4,415 \text { ) }\end{array}$ \\
\hline Age, y & $63.9 \pm 11.0$ \\
\hline Female & $2,426(55.0)$ \\
\hline White & $3,864(97.3)$ \\
\hline \multicolumn{2}{|l|}{ Smoking } \\
\hline Never & $1,367(31.0)$ \\
\hline Former & $2,120(48.0)$ \\
\hline Current & $928(21.0)$ \\
\hline Lower education & $1,266(28.7)$ \\
\hline Middle education & $2,107(47.7)$ \\
\hline Higher education & $1,042(23.6)$ \\
\hline Systolic blood pressure, $\mathrm{mm} \mathrm{Hg}$ & $140.0 \pm 21.5$ \\
\hline Diastolic blood pressure, $\mathrm{mm} \mathrm{Hg}$ & $83.2 \pm 10.9$ \\
\hline Antihypertensive medication & $1,573(35.6)$ \\
\hline Total cholesterol, mmol/L & $5.5 \pm 1.1$ \\
\hline HDL cholesterol, mmol/L & $1.5 \pm 0.4$ \\
\hline Lipid-lowering medication & $1,113(25.2)$ \\
\hline Diabetes mellitus & $531(12.0)$ \\
\hline BMI, kg/m² & $27.4 \pm 4.1$ \\
\hline CES-D & $8(2-12)$ \\
\hline$A P O E$ \& 4 carriership & $1,216(28.3)$ \\
\hline FA & $0.34 \pm 0.02$ \\
\hline MD & $0.74 \pm 0.03$ \\
\hline Intracranial volume, mL & $1,142.0 \pm 116.4$ \\
\hline White matter volume, $\mathrm{mL}$ & $409.3 \pm 60.7$ \\
\hline WMHs volume, $\mathrm{mL}$ & $2.90(1.6-6.3)$ \\
\hline
\end{tabular}

Abbreviations: $\mathrm{BMI}=$ body mass index; CES-D = Center for Epidemiologic Studies Depression Scale; FA = fractional anisotropy; $\mathrm{HDL}=$ high-density lipoprotein; $\mathrm{MD}=$ mean diffusivity $\times 10^{-3} \mathrm{~mm}^{2} / \mathrm{s} ; \mathrm{WMH}=$ white matter hyperintensity.

Continuous variables are presented as mean \pm SD and categorical variables as n (\%), except for WMHs volume and CES-D score, which are presented as median (interquartile range).

In tract-specific analyses, the strongest associations with dementia risk were observed for $\mathrm{MD}$ in the projection tracts, association tracts, and limbic system tracts (per SD increase HR of 2.35 [1.53-3.62] for the superior thalamic radiation, 1.79 [1.36-2.37] for the inferior fronto-occipital fasciculus, and 1.62 [1.41-1.86] for the parahippocampal part of the cingulum, respectively; table 3 and figure 2). Similarly, lower FA in the association tracts and in the limbic system tracts were most profoundly associated with a higher risk of dementia (per SD increase HR 0.59 [0.45-0.76] for the uncinated fasciculus and HR 0.67 [0.53-0.84] for the parahippocampal part of the cingulum, respectively, in the fully adjusted model; table 3). Similar patterns were seen for a clinical diagnosis of $\mathrm{AD}$ only (data available from Dryad, table e-2, 10.5061/dryad.7wm37pvpq).

The association between global white matter microstructure and cognitive decline is presented in table 4 . Higher values of global MD were associated with greater decline in global cognition, driven by worse performance on the Word Fluency Test and Stroop reading and interference subtasks. Results were unaltered by exclusion of all incident dementia cases (table 5). Similar associations, albeit somewhat attenuated, were observed for FA.

\section{Discussion}

In this longitudinal population-based study, we found that structural disconnectivity is associated with increased risk of dementia and with more pronounced cognitive decline. These associations were most profound for the projection, association, and limbic system tracts, and extended into the preclinical phase of the disease.

Longitudinal studies provide higher evidence for causal relations. Our main results provide evidence for the disconnection hypothesis, which states that loss of brain connections precedes cognitive decline and dementia. In line with this hypothesis, our results suggest that disconnectivity plays a role already in the preclinical stages of dementia. The findings in this study also extend results from clinical studies in patients with cerebral small vessel disease to the general population, ${ }^{14-17}$ suggesting that measures of FA/MD may improve prognostic accuracy of existing prediction models to identify persons at high risk of dementia in the community. Furthermore, knowledge of tract-specific effects on cognition and risk of dementia may allow clinicians to better understand why specific patients with only small, but strategically located brain infarcts develop cognitive impairment, and which patients after stroke are most likely to develop dementia. $^{30,31}$

Various potential pathways could lead to disconnectivity. A vascular pathway has been proposed in which reduction in white matter perfusion, e.g., due to impaired autoregulation, may result in white matter damage. ${ }^{32}$ Oligodendrocytes might shrink because of hypoxia and ischemia in white matter, with subsequent loss of myelin. ${ }^{33,34}$ However, in our fully adjusted model, we corrected for several cardiovascular risk factors and the estimates did not change substantially. This may be explained by residual confounding (due to age-specific effects of vascular factors or subclinical vascular factors), or a more complex, multifaceted pathway, in which there is a complex interplay of traditional vascular risk factors, hypoxia, and neuroinflammation. ${ }^{35}$ Inflammation-induced 
Table 2 Global white matter microstructure and incident dementia

\begin{tabular}{|c|c|c|c|}
\hline & Model & FA & MD \\
\hline \multirow[t]{2}{*}{ All dementia $(n=101)$} & Model I & $0.65(0.53-0.80)^{\mathrm{a}}$ & $1.77(1.43-2.17)^{\mathrm{a}}$ \\
\hline & Model II & $0.65(0.52-0.80)^{a}$ & $1.79(1.44-2.23)^{\mathrm{a}}$ \\
\hline \multirow[t]{2}{*}{$A D(n=83)$} & Model I & $0.70(0.55-0.88)^{\mathrm{a}}$ & $1.71(1.35-2.16)^{\mathrm{a}}$ \\
\hline & Model II & $0.69(0.54-0.88)^{a}$ & $1.76(1.38-2.24)^{\mathrm{a}}$ \\
\hline \multirow[t]{2}{*}{ Censoring for stroke $(n=98)$} & Model I & $0.65(0.53-0.80)^{a}$ & $1.75(1.41-2.16)^{\mathrm{a}}$ \\
\hline & Model II & $0.64(0.52-0.80)^{a}$ & $1.76(1.42-2.20)^{\mathrm{a}}$ \\
\hline \multirow[t]{2}{*}{ Exclusion cortical infarcts $(n=97)$} & Model I & $0.63(0.51-0.78)^{a}$ & $1.75(1.41-2.17)^{\mathrm{a}}$ \\
\hline & Model II & $0.61(0.49-0.77)^{a}$ & $1.79(1.43-2.24)^{\mathrm{a}}$ \\
\hline
\end{tabular}

Abbreviations: $A D$ = Alzheimer disease; $F A$ = fractional anisotropy; MD = mean diffusivity.

Data are presented as hazard ratio (95\% confidence interval) per SD increase of FA and MD. Model I: adjusted for age, sex, education, intracranial volume, white matter volume, and the log-transformed white matter hyperintensity volume. Model II: model I and in addition adjusted for Center for Epidemiologic Studies Depression Scale score, cardiovascular risk factors (systolic blood pressure, diastolic blood pressure, antihypertensive medication, serum cholesterol, high-density lipoprotein cholesterol, lipid-lowering medication, diabetes, smoking, body mass index), and $A P O E$ \&4 allele carriership.

a Significant at $p<0.05$.

disconnectivity may be caused by inflammation-related cytokines (tumor necrosis factor- $\alpha$, interleukin-8, interleukin-10, interferon- $\gamma$ ) and growth factors (IGFBP2, PDGF-BB), which have been associated with a lower integrity of myelin sheaths. ${ }^{36,37}$ Yet reverse causality as an explanation for our findings is unlikely since the risk estimates did not change after excluding the first 5 years of follow-up. Also, disconnectivity associated with cognitive decline also in individuals who did not develop dementia during the study duration, suggesting an association already in the preclinical phase of dementia and with normal aging.

We found that structural disconnectivity, indicated by a low FA and high MD throughout the brain, but in particular in

Figure 1 Global mean diffusivity and incident dementia, with exclusion of the first 5 years of follow-up

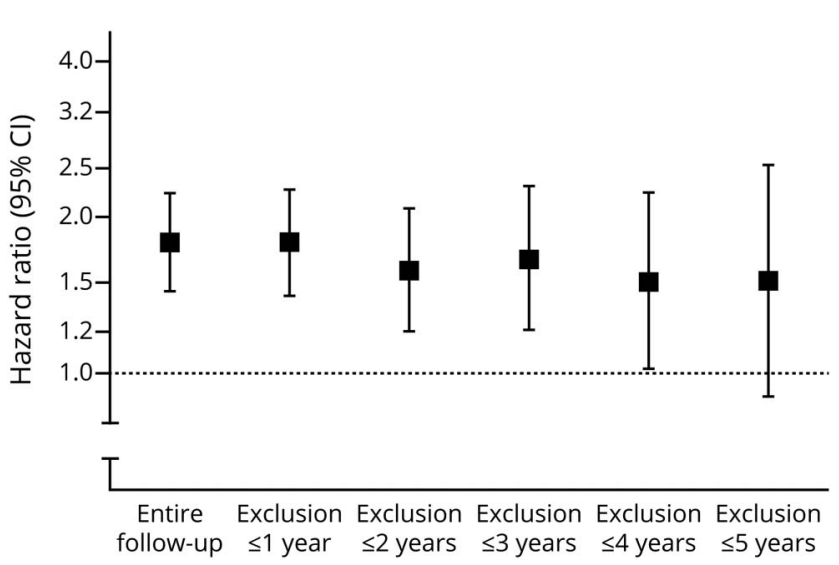

$\mathrm{Cl}=$ confidence interval. the projection, association, and limbic system tracts, related to a higher risk of dementia. This is in line with previous research in cross-sectional studies that found lower FA in white matter tracts including the association tracts ${ }^{38,39}$ and projection tracts ${ }^{40,41}$ associated with dementia. Lower FA values in limbic system tracts (in particular in the parahippocampal cingulum) and the association with dementia, more specifically $\mathrm{AD}$, has been most consistently reported in previous studies. $40,42,43$

A small number of studies reported higher FA values in specific regions in $\mathrm{AD} .^{44,45}$ This counterintuitive finding may be explained by selective degeneration of a fiber population in regions with crossing white matter tracts, leading to paradoxical higher FA. ${ }^{46} \mathrm{MD}$ is therefore thought to be a more sensitive and reliable measure in these crossing fiber regions (and therefore also globally), ${ }^{47}$ and presumably more sensitive to white matter damage. ${ }^{1,12}$ Moreover, in a small group of patients with $\mathrm{AD}$, increases in $\mathrm{MD}$ preceded changes in FA, which only occurred in a more progressive disease state. ${ }^{11}$ Accordingly, in our study we found stronger associations with MD than with FA.

The exact pathologic substrate underlying the changes in FA and MD leading to disconnectivity is unknown. There is pathologic evidence that changes in diffusion MRI measures correlate with myelin damage and axonal count, ${ }^{48}$ that myelin is increasingly suggested as an important factor in $\mathrm{AD}$ pathology, and that myelin breakdown is at the core of the earliest changes involved in dementia. ${ }^{49}$ However, the presence of other possible processes such as an increased water content in white matter due to loss of connectivity or inflammation generates difficulties in assigning change in diffusion MRI measures to a specific 
Table 3 Tract-specific white matter microstructure and incident dementia

\begin{tabular}{|c|c|c|c|c|}
\hline \multirow[b]{2}{*}{ White matter tracts } & \multicolumn{2}{|c|}{ Fractional anisotropy } & \multicolumn{2}{|l|}{ Mean diffusivity } \\
\hline & Model I & Model II & Model I & Model II \\
\hline \multicolumn{5}{|l|}{ Brainstem tracts } \\
\hline Middle cerebellar peduncle & $1.05(0.85-1.30)$ & $1.08(0.87-1.35)$ & $1.05(0.85-1.30)$ & $1.04(0.83-1.30)$ \\
\hline Medial lemniscus & $1.09(0.86-1.39)$ & $1.11(0.86-1.44)$ & $1.06(0.88-1.28)$ & $1.06(0.87-1.29)$ \\
\hline \multicolumn{5}{|l|}{ Projection tracts } \\
\hline Corticospinal tract & $1.17(0.95-1.44)$ & $1.19(0.96-1.47)$ & $1.52(1.13-2.06)^{a}$ & $1.52(1.11-2.08)^{a}$ \\
\hline Anterior thalamic radiation & $0.85(0.66-1.09)$ & $0.87(0.67-1.13)$ & $1.68(1.23-2.30)^{a, b}$ & $1.73(1.26-2.38)^{a, b}$ \\
\hline Superior thalamic radiation & $1.17(0.95-1.45)$ & $1.20(0.97-1.50)$ & $2.29(1.49-3.52)^{a, b}$ & $2.35(1.53-3.62)^{a, b}$ \\
\hline Posterior thalamic radiation & $0.69(0.52-0.90)^{a}$ & $0.74(0.56-0.97)^{a}$ & $1.41(1.15-1.72)^{a, b}$ & $1.42(1.15-1.75)^{a, b}$ \\
\hline \multicolumn{5}{|l|}{ Association tracts } \\
\hline Superior longitudinal fasciculus & $0.77(0.60-1.00)$ & $0.79(0.60-1.04)$ & $1.65(1.30-2.11)^{a, b}$ & $1.65(1.28-2.14)^{a, b}$ \\
\hline Inferior longitudinal fasciculus & $0.79(0.62-1.01)$ & $0.84(0.65-1.09)$ & $1.73(1.36-2.21)^{\mathrm{a}, \mathrm{b}}$ & $1.69(1.31-2.18)^{a, b}$ \\
\hline Inferior fronto-occipital fasciculus & $0.66(0.50-0.86)^{a, b}$ & $0.71(0.53-0.93)^{a}$ & $1.75(1.34-2.27)^{a, b}$ & $1.79(1.36-2.37)^{a, b}$ \\
\hline Uncinate fasciculus & $0.60(0.47-0.77)^{a, b}$ & $0.59(0.45-0.76)^{a, b}$ & $1.67(1.39-2.00)^{a, b}$ & $1.73(1.42-2.10)^{a, b}$ \\
\hline \multicolumn{5}{|l|}{ Limbic system tracts } \\
\hline Cingulate gyrus part of cingulum & $0.69(0.54-0.87)$ & $0.71(0.55-0.90)^{a}$ & $1.55(1.26-1.92)^{a, b}$ & $1.58(1.26-1.97)^{a, b}$ \\
\hline Parahippocampal part of cingulum & $0.67(0.54-0.84)^{a, b}$ & $0.67(0.53-0.84)^{a, b}$ & $1.61(1.41-1.85)^{a, b}$ & $1.62(1.41-1.86)^{a, b}$ \\
\hline Fornix & $0.76(0.59-0.99)^{a}$ & $0.78(0.60-1.02)$ & $1.13(0.80-1.58)$ & $1.06(0.75-1.50)$ \\
\hline \multicolumn{5}{|l|}{ Callosal tracts } \\
\hline Forceps major & $0.77(0.59-1.00)^{a}$ & $0.79(0.61-1.04)$ & $1.15(0.93-1.41)$ & $1.12(0.90-1.38)$ \\
\hline Forceps minor & $0.78(0.60-1.01)$ & $0.80(0.61-1.06)$ & $1.38(1.12-1.71)^{a}$ & $1.39(1.11-1.75)^{a}$ \\
\hline \multicolumn{5}{|c|}{$\begin{array}{l}\text { Data are presented as hazard ratio ( } 95 \% \text { confidence interval) per SD increase of fractional anisotropy and mean diffusivity. Model I: adjusted for age, sex, } \\
\text { education, intracranial volume, white matter volume, and the log-transformed white matter lesion volume of the investigated tract. Model II: model I and in } \\
\text { addition adjusted for Center for Epidemiologic Studies Depression Scale score, cardiovascular risk factors (systolic blood pressure, diastolic blood pressure, } \\
\text { antihypertensive medication, serum cholesterol, high-density lipoprotein cholesterol, lipid-lowering medication, diabetes, smoking, body mass index), and } \\
\text { APOE \&4 allele carriership. } \\
\text { a Significant at } p<0.05 \text {. } \\
\text { b Significant at } p<0.0029 \text {. }\end{array}$} \\
\hline
\end{tabular}

underlying pathologic process causing the observed structural disconnectivity. ${ }^{50,51}$

Strengths of the study are the population-based setting, the large sample size, the automated publicly available diffusion MRI processing methods that facilitate replication, ${ }^{8}$ and the longitudinal assessment of cognitive performance with meticulous follow-up for dementia. Some limitations need to be considered. First, the averaging of FA and MD measures over the normal-appearing white matter for analyses discards some spatial information. Second, given the long preclinical phase of dementia, our median follow-up time of 6.8 years is still relatively short, and longer duration studies with repeated imaging are required to further map changes in diffusion MRI in the process of neurodegeneration. Nevertheless, our results were unaffected by excluding the first 5 years of follow-up and independent of macrostructural white matter pathology (i.e., WMH volume). Third, although we found associations similar for allcause dementia and clinical $\mathrm{AD}$, confirmation of subtype diagnosis by (CSF) biomarkers or pathologic examination was not available and clinical diagnosis of $\mathrm{AD}$ has a low specificity for $\mathrm{AD}$ pathology. Fourth, we cannot rule out some partial volume effects by CSF contamination driving the observed change in diffusion metrics. Fifth, depression and vascular factors were assessed at baseline only, and some residual confounding by changes over time cannot be excluded.

Structural disconnectivity increases the risk of dementia and more pronounced cognitive decline. Our study suggests that diffusion MRI may be useful in risk prediction. 
Figure 2 Tract-specific microstructural integrity and incident dementia

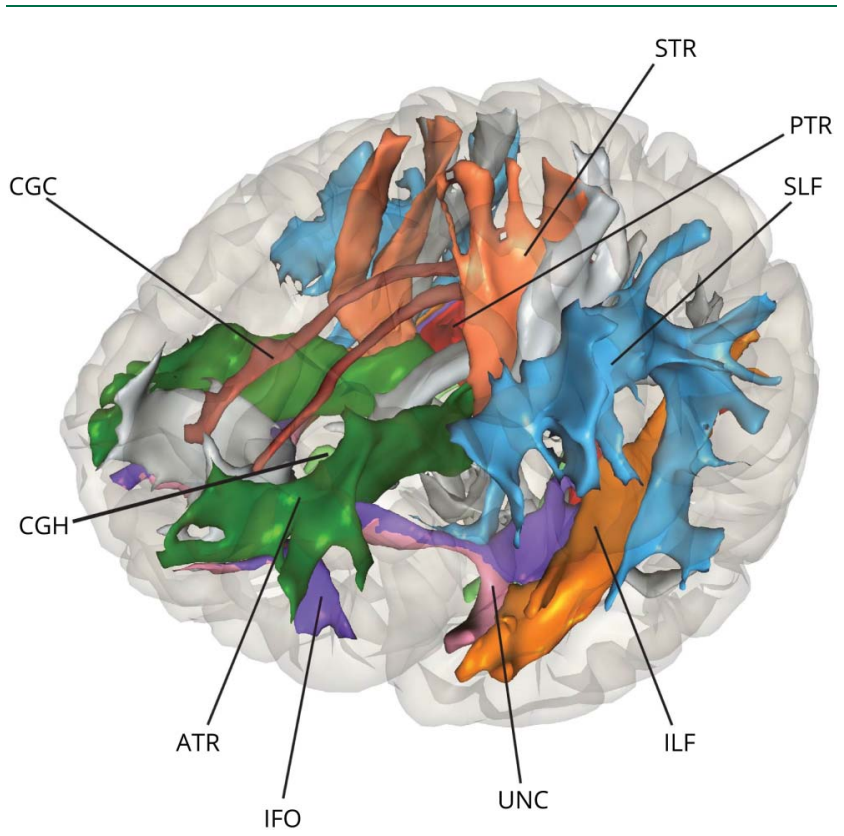

Tracts that were significantly associated with dementia risk are color-coded. Other tracts are presented in gray. ATR = anterior thalamic radiation; CGC = cingulate gyrus part of cingulum; $\mathrm{CGH}=$ parahippocampal part of cingulum; IFO = inferior-fronto-occipital fasciculus; ILF = inferior longitudinal fasciculus; PTR = posterior thalamic radiation; SLF = superior longitudinal fasciculus; STR = superior thalamic radiation; UNC = uncinate fasciculus.

\section{Acknowledgment}

The authors thank the staff at the Rotterdam Study research center and Frank J.A. van Rooij, data manager.

\section{Study funding}

Funding was obtained from the Internationale Stichting Alzheimer Onderzoek 12533, European Union Seventh Framework Programma (FP7/2007-2013) under grant agreement 601055, VPH-Dare@IT （FP7-ICT-2011-9601055) and the STW perspectief programme Population Imaging Genetics (ImaGene) projects 12722 and 12723, supported by the Dutch Technology Foundation STW, which is part of the Netherlands Organisation for scientific research (NWO) and partly funded by the Dutch Ministry of Economic Affairs. None of the funding sources influenced design or conduct of the study; collection, management, analysis, or interpretation of the data; preparation, review, or approval of the manuscript; or decision to submit the manuscript for publication.

\section{Disclosure}

L.G.M. Cremers, F.J. Wolters, M. de Groot, M. Kamran Ikram, and A. Van der Lugt report no disclosures relevant to the manuscript. W.J. Niessen is cofounder, shareholder, and CSO of Quantib BV. M.W. Vernooij reports grants from the Internationale Stichting Alzheimer Onderzoek 12533, European Union Seventh Framework Programma (FP7/20072013) under grant agreement 601055, VPH-Dare@IT (FP7-ICT-2011-9-601055), and the STW perspectief programme Population Imaging Genetics (ImaGene) projects 12722 and 12723, supported by the Dutch Technology Foundation STW, which is part of the Netherlands Organisation for Scientific Research (NWO) and partly funded by the Dutch Ministry of Economic Affairs, during the conduct of the study. M. Arfan Ikram reports no disclosures relevant to the manuscript. Go to Neurology.org/ $\mathrm{N}$ for full disclosures.

Table 4 Global white matter microstructure and cognitive decline

\begin{tabular}{llr}
\hline & FA & MD \\
\hline G-factor & $0.02(-0.004$ to 0.041$)$ & $-0.04(-0.07 \text { to }-0.01)^{\mathrm{a}}$ \\
\hline Immediate recall & $-0.002(-0.04$ to 0.03$)$ & $-0.03(-0.07$ to 0.02$)$ \\
\hline Delayed recall & $0.007(-0.03$ to 0.04$)$ & $-0.03(-0.07$ to -0.01$)$ \\
\hline Stroop reading task & $0.04(0.01 \text { to } 0.07)^{\mathrm{a}, \mathrm{b}}$ & $-0.06(-0.09 \text { to }-0.02)^{\mathrm{a}, \mathrm{b}}$ \\
\hline Stroop color naming task & $0.02(-0.001$ to 0.05$)$ & $-0.02(-0.05$ to 0.02$)$ \\
\hline Stroop interference task & $0.04(0.01 \text { to } 0.07)^{\mathrm{a}, \mathrm{b}}$ & $-0.09(-0.12 \text { to }-0.05)^{\mathrm{a}, \mathrm{b}}$ \\
\hline Letter-digit substitution task & $0.004(-0.02$ to 0.03$)$ & $-0.004(-0.04$ to 0.03$)$ \\
\hline Word fluency test & $0.03(0.001$ to 0.06$)$ & $-0.06(-0.10 \text { to }-0.02)^{\mathrm{a}, \mathrm{b}}$ \\
\hline Purdue pegboard & $0.03(0.005 \text { to } 0.06)^{\mathrm{a}}$ & $-0.04(-0.07$ to -0.00$)$ \\
\hline
\end{tabular}

Abbreviations: $F A$ = fractional anisotropy; $M D=$ mean diffusivity.

Data are presented as mean difference in $Z$ score ( $95 \%$ confidence interval) per SD increase of FA and MD.

a Significant at $p<0.05$.

b Significant at $p<0.008$.

Model adjusted for age, sex, education, intracranial volume, white matter volume, the log-transformed white matter lesion volume, Center for Epidemiologic Studies Depression Scale score, and in addition adjusted for cardiovascular risk factors (systolic blood pressure, diastolic blood pressure, antihypertensive medication, serum cholesterol, high-density lipoprotein cholesterol, lipid-lowering medication, diabetes, smoking, body mass index) and APOE \&4 allele carriership. 
Table 5 Global white matter microstructural integrity and cognitive decline (after exclusion of all incident dementia cases)

\begin{tabular}{llr}
\hline & FA & MD \\
\hline G-factor & $0.01(-0.01$ to 0.04$)$ & $-0.03(-0.06 \text { to }-0.001)^{\mathrm{a}}$ \\
\hline Immediate recall & $-0.007(-0.04$ to 0.03$)$ & $-0.01(-0.06$ to 0.03$)$ \\
\hline Delayed recall & $0.002(-0.03$ to 0.03$)$ & $-0.02(-0.06$ to 0.02$)$ \\
\hline Stroop reading task & $0.04(0.01 \text { to } 0.07)^{\mathrm{a}, \mathrm{b}}$ & $-0.06(-0.09 \text { to }-0.02)^{\mathrm{a}, \mathrm{b}}$ \\
\hline Stroop color naming task & $0.03(0.003 \text { to } 0.05)^{\mathrm{a}}$ & $-0.02(-0.05$ to -0.01$)$ \\
\hline Stroop interference task & $0.04(0.009 \text { to } 0.06)^{\mathrm{a}}$ & $-0.08(-0.12 \text { to }-0.04)^{\mathrm{a}, \mathrm{b}}$ \\
\hline Letter-digit substitution task & $0.005(-0.02$ to 0.03$)$ & $-0.004(-0.04$ to 0.02$)$ \\
\hline Word fluency test & $0.03(0.002 \text { to } 0.06)^{\mathrm{a}}$ & $-0.06(-0.10 \text { to }-0.02)^{\mathrm{a}, \mathrm{b}}$ \\
\hline Purdue pegboard & $0.03(0.002 \text { to } 0.06)^{\mathrm{a}}$ & $-0.03(-0.06$ to 0.009$)$ \\
\hline
\end{tabular}

Abbreviations: $\mathrm{FA}=$ fractional anisotropy; $\mathrm{MD}=$ mean diffusivity.

Data are presented as mean difference in $Z$ score (95\% confidence interval) per SD increase of FA and MD.

a Significant at $p<0.05$.

b Significant at $p<0.008$.

Model adjusted for age, sex, education, intracranial volume, white matter volume, and the log-transformed white matter hyperintensity volume, and in addition adjusted for Center for Epidemiologic Studies Depression Scale score, cardiovascular risk factors (systolic blood pressure, diastolic blood pressure, antihypertensive medication, serum cholesterol, high-density lipoprotein cholesterol, lipid-lowering medication, diabetes, smoking, body mass index), and $A P O E$ \&4 allele carriership.

\section{Publication history}

Received by Neurology July 29, 2019. Accepted in final form March 18, 2020.

Appendix Authors

\begin{tabular}{ll}
\hline Name & Location \\
\hline Lotte & Departments of Radiology \\
G.M. & and Epidemiology, Erasmus \\
Cremers, & MC, Rotterdam, the \\
PhD & Netherlands
\end{tabular}

\section{Contribution}

Collected and analyzed the data, interpreted the results, drafted the manuscript, had full access to all the data in the study and takes responsibility for the integrity of the data and the accuracy of the data analysis

\begin{tabular}{ll}
\hline $\begin{array}{l}\text { Frank J. } \\
\text { Wolters, } \\
\text { MD }\end{array}$ & $\begin{array}{l}\text { Departments of } \\
\text { Epidemiology, Radiology, } \\
\text { and Neurology, Erasmus MC, } \\
\text { Rotterdam, the Netherlands }\end{array}$ \\
\hline $\begin{array}{l}\text { Marius } \\
\text { de Groot, } \\
\text { PhD }\end{array}$ & $\begin{array}{l}\text { Departments of Radiology, } \\
\text { Epidemiology, and Medical } \\
\text { Informatics, Erasmus MC, } \\
\text { Rotterdam, the Netherlands }\end{array}$ \\
$\begin{array}{l}\text { M. } \\
\text { Kamran } \\
\text { Ikram, } \\
\text { PhD }\end{array}$ & $\begin{array}{l}\text { Departments of Neurology } \\
\text { and Epidemiology, Erasmus }\end{array}$ \\
$\begin{array}{l}\text { MC, Rotterdam, the } \\
\text { Netherlands }\end{array}$
\end{tabular}

Collected and analyzed the data, interpreted the results, drafted the manuscript

Collected the data, interpreted the results, drafted the manuscript

Designed and conceptualized the study, acquired funding, supervised the study, revised the manuscript for intellectual content

\begin{tabular}{ll}
\hline Aad van & Department of Radiology, \\
der Lugt, & Erasmus MC, Rotterdam, the \\
PhD & Netherlands
\end{tabular}

Designed and conceptualized the study, acquired funding, supervised the study, revised the manuscript for intellectual content
Appendix (continued)

\begin{tabular}{lll}
\hline Name & Location & Contribution \\
\hline $\begin{array}{l}\text { Wiro J. } \\
\text { Niessen, } \\
\text { PhD }\end{array}$ & $\begin{array}{l}\text { Departments of Radiology } \\
\text { and Medical Informatics, } \\
\text { Erasmus MC, Rotterdam; } \\
\text { Department of Imaging } \\
\begin{array}{l}\text { Physics, Delft University of } \\
\text { Technology, the Netherlands }\end{array}\end{array}$ & $\begin{array}{l}\text { Designed and } \\
\text { conceptualized the study, } \\
\text { acquired funding, } \\
\text { supervised the study, } \\
\text { revised the manuscript for } \\
\text { intellectual content }\end{array}$ \\
\hline $\begin{array}{l}\text { Meike W. } \\
\text { Vernooij, } \\
\text { PhD }\end{array}$ & $\begin{array}{l}\text { Departments of Radiology } \\
\text { and Epidemiology, Erasmus } \\
\text { MC, Rotterdam, the }\end{array}$ & $\begin{array}{l}\text { Designed and } \\
\text { conceptualized the study, } \\
\text { interpreted the results, }\end{array}$ \\
& Netherlands & $\begin{array}{l}\text { acquired funding, } \\
\text { supervised the study, } \\
\text { revised the manuscript for } \\
\text { intellectual content }\end{array}$ \\
\hline $\begin{array}{l}\text { M. Arfan } \\
\text { Ikram, } \\
\text { PhD }\end{array}$ & $\begin{array}{l}\text { Department of } \\
\text { Epidemiology, Erasmus MC, } \\
\text { Rotterdam, the Netherlands }\end{array}$ & $\begin{array}{l}\text { Designed and } \\
\text { conceptualized the study, } \\
\text { interpreted the results, } \\
\text { acquired funding, } \\
\text { supervised the study, } \\
\text { revised the manuscript for } \\
\text { intellectual content }\end{array}$ \\
\hline
\end{tabular}

\section{References}

1. Ferri CP, Prince M, Brayne C, et al. Global prevalence of dementia: a Delphi consensus study. Lancet 2005;366:2112-2117.

2. Jack CR Jr, Knopman DS, Jagust WJ, et al. Hypothetical model of dynamic biomarker of the Alzheimer's pathological cascade. Lancet Neurol 2010;9:119-128.

3. Petersen RC. How early can we diagnose Alzheimer disease (and is it sufficient)? The 2017 Wartenberg lecture. Neurology 2018;91:395-402.

4. Licher S, Leening MJG, Yilmaz P, et al. Development and validation of a dementia risk prediction model in the general population: an analysis of three longitudinal studies. Am J Psychiatry 2019;176:543-551.

5. Reid AT, Evans AC. Structural networks in Alzheimer's disease. Eur Neuropsychopharmacol 2013;23:63-77.

6. Daianu M, Dennis EL, Jahanshad N, et al. Alzheimer's disease disrupts rich club organization in brain connectivity networks. Proc IEEE Int Symp Biomed Imaging 2013:266-269. 
7. O'Sullivan M, Jones DK, Summers PE, et al. Evidence for "cortical disconnection" as a mechanism of age-related cognitive decline. Neurology 2001;57:632-638.

8. de Groot M, Verhaaren BF, de Boer R, et al. Changes in normal-appearing white matter precede development of white matter lesions. Stroke 2013;44:1037-1042.

9. Nazeri A, Chakravarty MM, Rajji TK, et al. Superficial white matter as a novel substrate of age-related cognitive decline. Neurobiol Aging 2015;36:2094-2106.

10. Vernooij MW, Ikram MA, Vrooman HA, et al. White matter microstructural integrity and cognitive function in a general elderly population. Arch Gen Psychiatry 2009;66: 545-553.

11. Acosta-Cabronero J, Alley S, Williams GB, Pengas G, Nestor PJ. Diffusion tensor metrics as biomarkers in Alzheimer's disease. PLoS One 2012;7:e49072.

12. Acosta-Cabronero J, Williams GB, Pengas G, Nestor PJ. Absolute diffusivities define the landscape of white matter degeneration in Alzheimer's disease. Brain 2010;133: 529-539.

13. Fu JL, Liu Y, Li YM, Chang C, Li WB. Use of diffusion tensor imaging for evaluating changes in the microstructural integrity of white matter over 3 years in patients with amnesic-type mild cognitive impairment converting to Alzheimer's disease. J Neuroimaging 2014;24:343-348.

14. Tuladhar AM, van Uden IW, Rutten-Jacobs LC, et al. Structural network efficiency predicts conversion to dementia. Neurology 2016;86:1112-1119.

15. Zeestraten EA, Lawrence AJ, Lambert C, et al. Change in multimodal MRI markers predicts dementia risk in cerebral small vessel disease. Neurology 2017;89: 1869-1876.

16. Tuladhar AM, Tay J, van Leijsen E, et al. Structural network changes in cerebral small vessel disease. J Neurol Neurosurg Psychiatry 2020;91:196-203.

17. Williams OA, Zeestraten EA, Benjamin P, et al. Predicting dementia in cerebral small vessel disease using an automatic diffusion tensor image segmentation technique. Stroke 2019;50:2775-2782.

18. Ikram MA, Brusselle GGO, Murad SD, et al. The Rotterdam Study: 2018 update on objectives, design and main results. Eur J Epidemiol 2017;32:807-850.

19. Ikram MA, van der Lugt A, Niessen WJ, et al. The Rotterdam Scan Study: design and update up to 2012. Eur J Epidemiol 2011;26:811-824.

20. Anbeek P, Vincken KL, van Bochove GS, van Osch MJ, van der Grond J. Probabilistic segmentation of brain tissue in MR imaging. Neuroimage 2005;27:795-804.

21. Vrooman HA, Cocosco CA, van der Lijn F, et al. Multi-spectral brain tissue segmentation using automatically trained k-nearest-neighbor classification. Neuroimage 2007;37:71-81.

22. de Boer R, Vrooman HA, van der Lijn F, et al. White matter lesion extension to automatic brain tissue segmentation on MRI. Neuroimage 2009;45:1151-1161.

23. Koppelmans V, de Groot M, de Ruiter MB, et al. Global and focal white matter integrity in breast cancer survivors 20 years after adjuvant chemotherapy. Hum Brain Mapp 2014;35:889-899.

24. de Groot M, Ikram MA, Akoudad S, et al. Tract-specific white matter degeneration in aging: the Rotterdam Study. Alzheimers Dement 2015;11:321-330.

25. de Bruijn RF, Bos MJ, Portegies ML, et al. The potential for prevention of dementia across two decades: the prospective, population-based Rotterdam Study. BMC Med $2015 ; 13: 132$.

26. Ligthart S, van Herpt TT, Leening MJ, et al. Lifetime risk of developing impaired glucose metabolism and eventual progression from prediabetes to type 2 diabetes: a prospective cohort study. Lancet Diabetes Endocrinol 2016;4:44-51.

27. Beekman AT, Deeg DJ, Van Limbeek J, et al. Criterion validity of the Center for Epidemiologic Studies Depression scale (CES-D): results from a community-based sample of older subjects in The Netherlands. Psychol Med 1997;27:231-235.

28. Galwey NW. A new measure of the effective number of tests, a practical tool for comparing families of non-independent significance tests. Genet Epidemiol 2009;33: 559-568.
29. Nyholt DR. A simple correction for multiple testing for single-nucleotide polymorphisms in linkage disequilibrium with each other. Am J Hum Genet 2004;74:765-769.

30. Zhao L, Biesbroek JM, Shi L, et al. Strategic infarct location for post-stroke cognitive impairment: a multivariate lesion-symptom mapping study. J Cereb Blood Flow Metab 2018;38:1299-1311.

31. Biesbroek JM, Leemans A, den Bakker $\mathrm{H}$, et al. Microstructure of strategic white matter tracts and cognition in memory clinic patients with vascular brain injury. Dement Geriatr Cogn Disord 2017;44:268-282.

32. Shi Y, Thrippleton MJ, Makin SD, et al. Cerebral blood flow in small vessel disease: a systematic review and meta-analysis. J Cereb Blood Flow Metab 2016;36:1653-1667.

33. Ihara M, Polvikoski TM, Hall R, et al. Quantification of myelin loss in frontal lobe white matter in vascular dementia, Alzheimer's disease, and dementia with Lewy bodies. Acta Neuropathol 2010;119:579-589.

34. Aboul-Enein F, Rauschka H, Kornek B, et al. Preferential loss of myelin-associated glycoprotein reflects hypoxia-like white matter damage in stroke and inflammatory brain diseases. J Neuropathol Exp Neurol 2003;62:25-33.

35. Heppner FL, Ransohoff RM, Becher B. Immune attack: the role of inflammation in Alzheimer disease. Nat Rev Neurosci 2015;16:358-372.

36. Benedetti F, Poletti S, Hoogenboezem TA, et al. Inflammatory cytokines influence measures of white matter integrity in Bipolar Disorder. J Affect Disord 2016;202:1-9.

37. Raj D, Yin Z, Breur M, et al. Increased white matter inflammation in aging- and Alzheimer's disease brain. Front Mol Neurosci 2017;10:206.

38. Stricker NH, Schweinsburg BC, Delano-Wood L, et al. Decreased white matter integrity in late-myelinating fiber pathways in Alzheimer's disease supports retrogenesis. Neuroimage 2009;45:10-16.

39. Damoiseaux JS, Smith SM, Witter MP, et al. White matter tract integrity in aging and Alzheimer's disease. Hum Brain Mapp 2009;30:1051-1059.

40. Mayo CD, Mazerolle EL, Ritchie L, et al. Longitudinal changes in microstructural white matter metrics in Alzheimer's disease. Neuroimage Clin 2017;13:330-338.

41. Serra L, Cercignani M, Lenzi D, et al. Grey and white matter changes at different stages of Alzheimer's disease. J Alzheimers Dis 2010;19:147-159.

42. Zhang Y, Schuff N, Jahng GH, et al. Diffusion tensor imaging of cingulum fibers in mild cognitive impairment and Alzheimer disease. Neurology 2007;68:13-19.

43. Kantarci K, Avula R, Senjem ML, et al. Dementia with Lewy bodies and Alzheimer disease: neurodegenerative patterns characterized by DTI. Neurology 2010;74: 1814-1821.

44. Teipel SJ, Grothe MJ, Filippi M, et al. Fractional anisotropy changes in Alzheimer's disease depend on the underlying fiber tract architecture: a multiparametric DTI study using joint independent component analysis. J Alzheimers Dis 2014;41:69-83.

45. Teipel SJ, Stahl R, Dietrich O, et al. Multivariate network analysis of fiber tract integrity in Alzheimer's disease. Neuroimage 2007;34:985-995.

46. Douaud G, Jbabdi S, Behrens TE, et al. DTI measures in crossing-fibre areas: increased diffusion anisotropy reveals early white matter alteration in MCI and mild Alzheimer's disease. Neuroimage 2011;55:880-890.

47. Jeurissen B, Leemans A, Tournier JD, Jones DK, Sijbers J. Investigating the prevalence of complex fiber configurations in white matter tissue with diffusion magnetic resonance imaging. Hum Brain Mapp 2013;34:2747-2766.

48. Beaulieu C. The basis of anisotropic water diffusion in the nervous system-a tech nical review. NMR Biomed 2002;15:435-455.

49. Bartzokis G. Age-related myelin breakdown: a developmental model of cognitive decline and Alzheimer's disease. Neurobiol Aging 2004;25:5-18; author reply 49-62.

50. Wood TC, Simmons C, Hurley SA, et al. Whole-brain ex-vivo quantitative MRI of the cuprizone mouse model. PeerJ 2016;4:e2632.

51. Duering M, Finsterwalder S, Baykara E, et al. Free water determines diffusion alterations and clinical status in cerebral small vessel disease. Alzheimers Dement 2018; 14 764-774. 